\title{
Association between sexual orientation and subjective cognitive complaints in the general population in England
}

\section{Introduction}

Subjective cognitive complaints (SCC) refer to everyday concerns cited by people both with and without objective evidence of cognitive impairment (Mitchell, 2008). A nationally representative study of adults from the UK showed that almost one third of the population reported forgetfulness in the last month, and that more than $6 \%$ had forgotten something important in the last week (Cooper et al., 2011). Another study reported that $30 \%$ of the unimpaired elderly claim to have "trouble remembering things that have happened recently" and a similar number have "trouble remembering where belongings are kept" (Jessen et al., 2007). This observed relatively high prevalence of SCC is of concern as SCC has been associated with detrimental health outcomes. For example, it is a core feature in mild cognitive impairment (MCI) (Mitchell, 2008), which is a preclinical state of dementia with a high conversion rate to dementia (Mitchell \& Shiri-Feshki, 2009). Moreover, one review concluded that older adults with memory complaints report a diminished quality of life (Mol et al., 2007). Other research has identified that SCC is also associated with mental disorders (Balash et al., 2013), and premature all-cause mortality (Ayalon, 2008).

Owing to the high prevalence of SCC and the detrimental health outcomes it is associated with, it is important to develop intervention and policy to aid in the prevention of SCC. In order to develop targeted interventions, correlates of SCC or vulnerable groups need to be identified. To date, several correlates of SCC have been identified. Specifically, these include physical multimorbidity (Jacob, Haro, \& Koyanagi, 2019), sleep problems (Miley-Akerstedt et al., 2018), 
older age (Newson \& Kemps, 2006), sex, personality traits, and menopause (Flatt et al., 2018), as well as affective symptoms (anxiety and depression) (Hill et al., 2016). More recently, literature has emerged suggesting that lesbian, gay, and bisexuals (i.e., sexual minorities) may be at a greater risk of SCC. For example, in a recent study of 210 sexual minority US adults (mean age 59.6 years, $\mathrm{SD}=8.0$ ), $25 \%$ of the sample were classified as having SCC (Flatt et al., 2018). Another study identified that up to $50 \%$ of sexual minority older adults reported moderate to severe SCC, and rates were higher among sexual minority older adults who were racial/ethnic minorities (Fredriksen-Goldsen, Jen, Bryan, \& Goldsen, 2018). There is a scarcity of other data to confirm or refute this finding and thus, there is a clear need for further research in this area.

Sexual minorities may be at greater risk of SCC for several reasons. When considering identified correlates of SCC, sexual minorities have been found to have high levels of sleep problems (Butler, McGlinchey, \& Juster, 2020), anxiety (Hayes-Skelton \& Pantalone, 2020) and stress (Dentato, 2012) due to experiences of prejudice, discrimination and victimization. It is therefore possible that such correlates may be associated with higher levels of SCC in sexual minorities.

Therefore, the aim of the current study was to investigate the association between identifying as a sexual minority and SCC in a large sample of adults from England. The study further aimed to identify the extent to which the association could be explained by several behavioral, psychological and clinical factors.

\section{Methods}

Study participants 
Data from the 2007 Adult Psychiatric Morbidity Survey (APMS) were used for this study. The survey has already been extensively described in the literature (Jenkins et al., 2009; NHS Digital, 2009). Succinctly, the APMS survey was a nationally representative survey of adults aged $\geq 16$ years living in private households in England. The survey fieldwork was undertaken by the National Center for Social Research and Leicester University between October 2006 and December 2007. A multistage stratified probability sampling design was used with the sampling frame consisting of the small user postcode address file and the primary sampling units corresponding to postcode sectors. Face-to-face interviews were conducted to obtain participant information, while some data were self-completed with the use of a computer. Sample weights were constructed to obtain a nationally representative sample, and these weights accounted for non-response and the probability of being selected. The response rate of the survey was $57 \%$. Finally, informed consent was obtained for all participants, and ethical permission for the study was obtained from the Royal Free Hospital and Medical School Research Ethics Committee.

\section{Measures}

\section{Sexual orientation (independent variable)}

Two items adapted from the Kinsey scale were used to assess sexual orientation: (a) "Which statement best describes your sexual orientation? This means sexual feelings, whether or not you have had any sexual partners." with answer options "entirely heterosexual", "mostly heterosexual", "bisexual", "mostly homosexual", "entirely homosexual”, and "other"; and (b) "Please choose the answer below that best describes how you currently think of yourself..." with answer options "completely heterosexual", "mainly heterosexual", "bisexual", "mainly gay or lesbian", “completely gay or lesbian", and "other". Initially, the aim of these two items was to investigate the impact of question wording and format on the proportion of sexual minority orientation in the overall population, and participants were therefore randomly 
allocated item (a) and item (b). In line with previous research (Jacob et al., 2019), we created a dichotomous variable on sexual orientation (heterosexual or sexual minority). Heterosexuality corresponded to "entirely heterosexual" - item (a) - or "completely heterosexual" - item (b) , while sexual minority identity was categorized by pooling to all other categories ("mostly heterosexual" - item (a) -, "bisexual" - item (a) -, "mostly homosexual" - item (a) -, "entirely homosexual" - item (a) -, "other" - item (a) -, "mainly heterosexual" - item (b) -, "bisexual" - item (b) -, "mainly gay or lesbian" - item (b) -, "completely gay or lesbian" - item (b) -, or "other" - item (b) -).

\section{Subjective cognitive complaints (dependent variables)}

SCC corresponded to subjective concentration and memory complaints. Following a previous publication (Jacob et al., 2019), two questions were used to assess these complaints: "In the past month, have you had any problems with concentrating on what you were doing?" (concentration) and "Have you noticed any problems with forgetting things in the past month?" (memory) with yes/no answers.

\section{Control variables}

Control variables were based on previous literature (Jacob et al., 2019) and included sex as assigned at birth (male and female), age (in years), ethnicity (British White: yes or no), marital status (single/separated/divorced/widowed or married/cohabiting), education (having a qualification [degree, non-degree, A-level, GCSE, other]: yes or no), employment (yes or no), and income (sex-equivalized income tertiles; high $\geq £ 29,826$, middle $£ 14,057-<£ 29,826$ and low $<£ 14,057)$.

\section{Influential factors}


Influential factors were variables that may play a mediating role in the association between sexual orientation and SCC. These influential factors included smoking status (McCabe et al., 2018; Sabia et al., 2012), alcohol dependence (Kim, Kim, \& Park, 2016; Pesola, Shelton, \& van den Bree, Marianne BM, 2014), perceived stress (Krueger, Meyer, \& Upchurch, 2018; Munoz, Sliwinski, Scott, \& Hofer, 2015), the number of stressful life events (Hatzenbuehler, Slopen, \& McLaughlin, 2014; Pilleron et al., 2015), depression (Semlyen, King, Varney, \& HaggerJohnson, 2016; Toyoshima et al., 2020), any anxiety disorder (Hill et al., 2016; Semlyen et al., 2016), sleep problems (Duncan et al., 2018; Tsapanou et al., 2019), and obesity (Azagba, Shan, \& Latham, 2019; Hou et al., 2019).

Smoking status. The following question "Have you ever smoked a cigarette?" was used to assess smoking status. Participants were dichotomized into never and past/current smokers.

Alcohol dependence. The Alcohol Use Disorders Identification Test (AUDIT) was used to screen excessive alcohol consumption (Saunders, Aasland, Babor, De la Fuente, Juan R, \& Grant, 1993), and alcohol dependence was assessed using the Severity of Alcohol Dependence Questionnaire (SADQ-C) in adults with an AUDIT score of $\geq 10$ (Stockwell, Sitharthan, McGRATH, \& Lang, 1994). Alcohol dependence in the past six months corresponded to SADQ-C scores $\geq 4$.

Perceived stress. Participants were asked if tasks at home and at work were stressful with answers ranging from 0 "not at all" to 3 "most of the time". Following previous research (Jacob et al., 2019), stress was dichotomized into "not at all" or "occasionally"/“usually"/“"most of the time".

Number of stressful life events. Stressful life events were assessed using 18 items (e.g., serious injury, death of a close family friend and violence at work), and the scale ranged from zero to 18. 
Depression and any anxiety disorder. The Clinical Interview Schedule Revised (CIS-R) was used to assess these common mental disorders in the prior week (Lewis, Pelosi, Araya, \& Dunn, 1992; National Centre for Social Research, 2007), and any anxiety disorder corresponded to generalized anxiety disorder, panic disorder, phobia, and obsessive-compulsive disorder.

Sleep problems. Sleep problems were assessed using two questions: "In the past month, have you been having problems with trying to get to sleep or with getting back to sleep if you woke up or were woken up?" (sleeping less than usual) and "Has sleeping more than you usually do been a problem for you in the past month?" (sleeping more than usual). Participants were considered to have sleep problems if they answered "yes" to at least one of these two questions. Obesity. Following the definition of the World Health Organization, obesity was defined as a body mass index $(\mathrm{BMI}) \geq 30 \mathrm{~kg} / \mathrm{m}^{2}$, with the BMI being calculated as self-reported weight (in kilograms) divided by squared self-reported height (in meters) (Jacob et al., 2019).

\section{Statistical analyses}

Given that the goal of this study was to focus on a cognitively healthy sample, the statistical analyses excluded four individuals who self-reported a doctor's diagnosis of Alzheimer's disease or dementia. Differences in terms of control variables and influential factors between heterosexual and sexual minority individuals were tested with Chi-squared tests for categorical variables and with Student's t-tests for continuous variables. In addition, the prevalence of subjective concentration and memory complaints was compared between the heterosexual and the sexual minority group using Chi-squared tests. The relationship of sexual orientation identity with subjective concentration and memory complaints was further studied in a base logistic regression model adjusted for sex, age, ethnicity, marital status, education, employment, and income. Subsequently, each of the eight influential factors (smoking status, alcohol dependence, perceived stress, number of stressful life events, depression, any anxiety 
disorder, sleep problems, and obesity) was individually included in this base model. Finally, all influential factors were simultaneously included in the base model (fully adjusted model). Percent attenuation in the log odds compared to the base model was estimated for all nine models. Results from the logistic regression analyses are presented as odds ratios (ORs) and 95\% confidence intervals (CIs). The sample weighting and the complex study design were taken into account in all analyses. The level of statistical significance was set at $p$-value $<0.05$. The statistical analyses were performed with R 3.6.2 (The R Foundation) (R Foundation for Statistical Computing, Vienna, Austria, 2019).

\section{Results}

This study included 7,400 participants (51.4\% women; mean [standard deviation] age 46.3 [18.6] years). The prevalence of identifying as as sexual minority was $7.1 \%$. Ethnicity other than British White, single/separated/divorced/widowed, alcohol dependence, perceived stress, depression, any anxiety disorder, and sleep disorders were more frequent in sexual minority individuals than in their heterosexual counterparts, while they were younger and had a higher number of stressful life events (Table 1). The prevalence of subjective concentration complaints was significantly higher in the sexual minority than in the heterosexual group (29.2\% versus $21.4 \%)$, whereas there was no significant difference in terms of subjective memory complaints (32.5\% versus $29.7 \%$; Figure 1). The results of the logistic regression analyses are displayed in Table $\mathbf{2}$. Identifying as a sexual minority was positively and significantly associated with subjective concentration $(\mathrm{OR}=1.40,95 \% \mathrm{CI}=1.12-1.76)$ but not memory complaints $(\mathrm{OR}=1.19,95 \% \mathrm{CI}=0.96-1.47)$ in the base model adjusted for sex, age, ethnicity, marital status, education, employment, and income. The number of stressful life events, sleep problems and any anxiety disorder explained $13.4 \%, 11.0 \%$ and $10.9 \%$ of the association between sexual orientation and subjective concentration complaints, respectively 
(these three variables explained together $24.8 \%$ of the association). When all influential factors were included in the logistic regression model (i.e., smoking status, alcohol dependence, perceived stress, number of stressful life events, depression, any anxiety disorder, sleep problems, and obesity), these influential factors collectively explained $29.6 \%$ of the relationship between sexual orientation identity and subjective concentration complaints.

\section{Discussion}

In this large sample of English adults, it was found that identifying as a sexual minority was associated with a 1.4 times higher odds of subjective concentration complaints and approximately $25 \%$ of this association was explained by stressful life events, sleep problems, and anxiety disorders. However, a non-significant association was found between sexual minority orientation and subjective memory complaints.

Our findings supports previous literature through confirming that sexual minorities are at a high risk of SCC (Flatt et al., 2018; Fredriksen-Goldsen et al., 2018) and adds to the literature by demonstrating that such an association also exists in a large sample from the UK. This study also supports previous finings showing that older sexual minorities are at higher risk of “memory complications" per se (Correro \& Nielson, 2020; Liu, Hsieh, Zhang, Zhang, \& Langa, 2020; Mayeux et al., 1993). Moreover, the present study shows for the first time that a large proportion of the association between sexual minority identity and SCC may be explained by stressful life events, sleep problems, and anxiety. Finally, this study advances the field by demonstrating that there is a more nuanced association in that identifying as a sexual minority is significantly associated with some aspects of SCC, but not all. 
However, only about $30 \%$ of the association between sexual orientation identities and SCC was explained by the influential factors assessed in our study. Thus, other factors not assessed in our study are also likely to explain the observed association. Firstly, it is widely recognized that sexual minorities are at increased risk of experiencing prejudice, discrimination and victimization. These experiences increase levels of stress among these populations and may contribute to poor sleep levels, increased anxiety and other mental health concerns. For example, in a recent study it was found that among sexual minority participants, discrimination based on age and sexual orientation predicted greater levels of mental health concerns (Lytle, Apriceno, Dyar, \& Levy, 2018). Secondly, sexual minorities, at least in the case for nonmonogamous men who have sex with men, are disproportionally impacted by HIV, which in itself is a risk factor for cognitive impairment and dementia (Heaton et al., 2011; Rourke, Halman, \& Bassel, 1999). It is possible that among these groups, a higher incidence of HIV diagnosis may help explain higher levels of SCC. Finally, among some communities, there may be a greater tendency to engage in some unfavorable lifestyle behaviours including, for example an, low levels of physical activity, particularly for those who identify as lesbian, or increased use of alcohol and narcotics, which have been shown to have a detrimental impact on cognition (Jedrziewski, Lee, \& Trojanowski, 2007).

It should be noted that although identifying as a sexual minority was associated with concentration complaints, it was non-significantly associated with memory complaints. The reason for the weaker association between sexual minority orientation and memory complaints and not concentration complaints is elusive and further research is needed to understand these nuanced findings. 
Findings from the present study and others (Flatt et al., 2018) suggest that it may be prudent to develop intervention and policy to prevent SCC among those who identify as a sexual minority. Such strategies should focus on increasing resilience to stressful life events, improving sleep, and reducing anxiety since these explained a large proportion of the observed association between sexual orientation and SCC. Although there are pharmacological treatments for sleep problems and anxiety, changes in lifestyle factors may also be important. For example, the promotion of physical activity has been shown to improve stress (Mayo Clinic Staff, 2020), sleep status (National Sleep Foundation, 2020), and reduce anxiety symptoms (McDowell, Dishman, Gordon, \& Herring, 2019). Therefore, it is possible that the promotion of physical activity among sexual minority groups may lead to reduction in SCC. Specifically, interventions that contain mind-body exercises (e.g. tai-chi, yoga) may result in favorable outcomes in relation to all mediators identified in the present study (Abbott \& Lavretsky, 2013; Chen et al., 2010; Kirkwood, Rampes, Tuffrey, Richardson, \& Pilkington, 2005).

The investigation of multiple domains of SCC and the large sample of English adults are clear strengths of the present study. However, findings must be interpreted in the light of several limitations. First, sexual orientation was assessed with two different items, and this may have impacted the findings of this study. Second, SCC included subjective concentration and memory complaints only, and a more complete measure may have allowed for more detailed analyses. Third, The APMS survey was conducted in 2007, and it is possible that the association between sexual orientation and SCC has changed in recent years. It is also possible that this association may be stronger during the current COVID-19 pandemic where people are generally suffering higher levels of mental health complications (Smith et al., 2020), lower levers of physical activity (Meyer et al., 2020), and an increase in alcohol consumption (Jacob et al., 2020). Future epidemiological studies are encouraged to collect data on sexual orientation and 
SCC. Fourth, the exposure and outcome were self-reported introducing self-reporting bias into our findings. Finally, this was a cross-sectional study, and it was therefore not possible to determine causality or temporality in the sexual orientation-SCC relationship. However, it is highly unlikely that SCC would lead to a change in sexual orientation.

In conclusion, in this large sample of English adults, identifying as a sexual minority was significantly associated with subjective concentration complaints but not memory complains. Stress, sleep problems, and anxiety explained a large proportion of the association between sexual orientation and concentration complaints. Targeted interventions towards sexual minority groups to reduce SCC may be warranted. Such interventions may wish to focus on mediators identified in the present study and thus implement interventions that focus on reducing stress, anxiety and improving sleep. Interventions that contain mind-body exercises (e.g. tai-chi, yoga) may result in favorable outcomes in relation to all mediators identified in the present study (Abbott \& Lavretsky, 2013; Chen et al., 2010; Kirkwood et al., 2005). 


\section{References}

Abbott, R., \& Lavretsky, H. (2013). Tai chi and qigong for the treatment and prevention of mental disorders. The Psychiatric Clinics of North America, 36(1), 109.

Ayalon, L. (2008). Subjective cognitive functioning as a predictor of all cause mortality in an israeli national sample of community dwelling older adults. International Journal of Geriatric Psychiatry, 23(8), 830-836.

Azagba, S., Shan, L., \& Latham, K. (2019). Overweight and obesity among sexual minority adults in the united states. International Journal of Environmental Research and Public Health, 16(10), 1828.

Balash, Y., Mordechovich, M., Shabtai, H., Giladi, N., Gurevich, T., \& Korczyn, A. D. (2013). Subjective memory complaints in elders: Depression, anxiety, or cognitive decline? Acta Neurologica Scandinavica, 127(5), 344-350.

Butler, E. S., McGlinchey, E., \& Juster, R. (2020). Sexual and gender minority sleep: A narrative review and suggestions for future research. Journal of Sleep Research, 29(1), e12928.

Chen, K., Chen, M., Lin, M., Fan, J., Lin, H., \& Li, C. (2010). Effects of yoga on sleep quality and depression in elders in assisted living facilities. Journal of Nursing Research, 18(1), 53-61.

Cooper, C., Bebbington, P., Lindesay, J., Meltzer, H., McManus, S., Jenkins, R., \& Livingston, G. (2011). The meaning of reporting forgetfulness: A cross-sectional study of adults in the english 2007 adult psychiatric morbidity survey. Age and Ageing, 40(6), 711-717. 
Correro, A. N., \& Nielson, K. A. (2020). A review of minority stress as a risk factor for cognitive decline in lesbian, gay, bisexual, and transgender (LGBT) elders. Journal of Gay \& Lesbian Mental Health, 24(1), 2-19.

Dentato, M. P. (2012). The minority stress perspective. Retrieved from https://www.apa.org/pi/aids/resources/exchange/2012/04/minority-stress

Duncan, D. T., Kanchi, R., Tantay, L., Hernandez, M., Letamendi, C., Chernov, C., \& Thorpe, L. (2018). Disparities in sleep problems by sexual orientation among new york city adults: An analysis of the new york city health and nutrition examination survey, 2013-2014. Journal of Urban Health, 95(6), 781-786.

Flatt, J. D., Johnson, J. K., Karpiak, S. E., Seidel, L., Larson, B., \& Brennan-Ing, M. (2018). Correlates of subjective cognitive decline in lesbian, gay, bisexual, and transgender older adults. Journal of Alzheimer's Disease, 64(1), 91-102.

Fredriksen-Goldsen, K. I., Jen, S., Bryan, A. E., \& Goldsen, J. (2018). Cognitive impairment, alzheimer's disease, and other dementias in the lives of lesbian, gay, bisexual and transgender (LGBT) older adults and their caregivers: Needs and competencies. Journal of Applied Gerontology, 37(5), 545-569.

Hatzenbuehler, M. L., Slopen, N., \& McLaughlin, K. A. (2014). Stressful life events, sexual orientation, and cardiometabolic risk among young adults in the united states. Health Psychology, 33(10), 1185.

Hayes-Skelton, S. A., \& Pantalone, D. W. (2020). Anxiety and depression in sexual and gender minority individuals. Retrieved from https://adaa.org/sexual-gender-minority$\underline{\text { individuals }}$ 
Heaton, R. K., Franklin, D. R., Ellis, R. J., McCutchan, J. A., Letendre, S. L., LeBlanc, S., . . . Woods, S. P. (2011). HIV-associated neurocognitive disorders before and during the era of combination antiretroviral therapy: Differences in rates, nature, and predictors. Journal of Neurovirology, 17(1), 3-16.

Hill, N. L., Mogle, J., Wion, R., Munoz, E., DePasquale, N., Yevchak, A. M., \& Parisi, J. M. (2016). Subjective cognitive impairment and affective symptoms: A systematic review. The Gerontologist, 56(6), e109-e127.

Hou, Q., Guan, Y., Yu, W., Liu, X., Wu, L., Xiao, M., \& Lü, Y. (2019). Associations between obesity and cognitive impairment in the chinese elderly: An observational study. Clinical Interventions in Aging, 14, 367.

Jacob, L., Haro, J. M., \& Koyanagi, A. (2019). Physical multimorbidity and subjective cognitive complaints among adults in the United Kingdom: A cross-sectional community-based study. Scientific Reports, 9(1), 1-11.

Jacob, L., Smith, L., Armstrong, N. C., Yakkundi, A., Barnett, Y., Butler, L., . . Meyer, J. (2020). Alcohol use and mental health during COVID-19 lockdown: A cross-sectional study in a sample of UK adults. Drug and Alcohol Dependence, 219, 108488.

Jacob, L., Smith, L., McDermott, D., Haro, J. M., Stickley, A., \& Koyanagi, A. (2019). Relationship between sexual orientation and psychotic experiences in the general population in England. Psychological Medicine, 1-9.

Jedrziewski, M. K., Lee, V. M., \& Trojanowski, J. Q. (2007). Physical activity and cognitive health. Alzheimer's \& Dementia, 3(2), 98-108. 
Jenkins, R., Meltzer, H., Bebbington, P., Brugha, T., Farrell, M., McManus, S., \& Singleton, N. (2009). The British Mental Health Survey Programme: Achievements and Latest Findings. Social Psychiatry and Psychiatric Epidemiology, 44, 899-904.

Jessen, F., Wiese, B., Cvetanovska, G., Fuchs, A., Kaduszkiewicz, H., Kölsch, H., . . . Bickel, H. (2007). Patterns of subjective memory impairment in the elderly: Association with memory performance. Psychological Medicine, 37(12), 1753-1762.

Kim, S., Kim, Y., \& Park, S. M. (2016). Association between alcohol drinking behaviour and cognitive function: Results from a nationwide longitudinal study of south korea. BMJ Open, 6(4).

Kirkwood, G., Rampes, H., Tuffrey, V., Richardson, J., \& Pilkington, K. (2005). Yoga for anxiety: A systematic review of the research evidence. British Journal of Sports Medicine, 39(12), 884-891.

Krueger, E. A., Meyer, I. H., \& Upchurch, D. M. (2018). Sexual orientation group differences in perceived stress and depressive symptoms among young adults in the united states. LGBT Health, 5(4), 242-249.

Lewis, G., Pelosi, A. J., Araya, R., \& Dunn, G. (1992). Measuring psychiatric disorder in the community: A standardized assessment for use by lay interviewers. Psychological Medicine, 22(2), 465-486.

Liu, H., Hsieh, N., Zhang, Z., Zhang, Y., \& Langa, K. M. (2020). Same-sex couples and cognitive impairment: Evidence from the health and retirement study. The Journals of Gerontology: Series B, gbaa202. 
Lytle, A., Apriceno, M., Dyar, C., \& Levy, S. R. (2018). Sexual orientation and gender differences in aging perceptions and concerns among older adults. Innovation in Aging, 2(3), igy036.

Mayeux, R., Stern, Y., Tang, M. X., Todak, G., Marder, K., Sano, M., . . Gorman, J. M. (1993). Mortality risks in gay men with human immunodeficiency virus infection and cognitive impairment. Neurology, 43(1 Part 1), 176.

Mayo Clinic Staff. (2020). Exercise and stress: Get moving to manage stress. Retrieved from https://www.mayoclinic.org/healthy-lifestyle/stress-management/in-depth/exercise-and$\underline{\text { stress/art-20044469 }}$

McCabe, S. E., Matthews, A. K., Lee, J. G., Veliz, P., Hughes, T. L., \& Boyd, C. J. (2018). Tobacco use and sexual orientation in a national cross-sectional study: Age, race/ethnicity, and sexual identity-attraction differences. American Journal of Preventive Medicine, 54(6), 736-745.

McDowell, C. P., Dishman, R. K., Gordon, B. R., \& Herring, M. P. (2019). Physical activity and anxiety: A systematic review and meta-analysis of prospective cohort studies. American Journal of Preventive Medicine, 57(4), 545-556.

Meyer, J., McDowell, C., Lansing, J., Brower, C., Smith, L., Tully, M., \& Herring, M. (2020). Changes in physical activity and sedentary behavior in response to COVID-19 and their associations with mental health in 3052 US adults. International Journal of Environmental Research and Public Health, 17(18), 6469.

Miley-Akerstedt, A., Jelic, V., Marklund, K., Walles, H., Åkerstedt, T., Hagman, G., \& Andersson, C. (2018). Lifestyle factors are important contributors to subjective memory 
complaints among patients without objective memory impairment or positive neurochemical biomarkers for alzheimer's disease. Dementia and Geriatric Cognitive Disorders Extra, 8(3), 439-452.

Mitchell, A. J. (2008). Is it time to separate subjective cognitive complaints from the diagnosis of mild cognitive impairment? Age and Ageing, 37(5), 497-499.

Mitchell, A. J., \& Shiri-Feshki, M. (2009). Rate of progression of mild cognitive impairment to dementia-meta-analysis of 41 robust inception cohort studies. Acta Psychiatrica Scandinavica, 119(4), 252-265.

Mol, M., Carpay, M., Ramakers, I., Rozendaal, N., Verhey, F., \& Jolles, J. (2007). The effect of perceived forgetfulness on quality of life in older adults; a qualitative review. International Journal of Geriatric Psychiatry: A Journal of the Psychiatry of Late Life and Allied Sciences, 22(5), 393-400.

Munoz, E., Sliwinski, M. J., Scott, S. B., \& Hofer, S. (2015). Global perceived stress predicts cognitive change among older adults. Psychology and Aging, 30(3), 487.

National Centre for Social Research. (2007). Adult psychiatric morbidity survey 2007. Retrieved from http://doc.ukdataservice.ac.uk/doc/6379/mrdoc/pdf/6379_apms_2007_dataset_document ation.pdf

National Sleep Foundation. (2020). How exercise impacts sleep quality. Retrieved from https://www.sleepfoundation.org/articles/how-exercise-impacts-sleep-quality

Newson, R. S., \& Kemps, E. B. (2006). The nature of subjective cognitive complaints of older adults. The International Journal of Aging and Human Development, 63(2), 139-151. 
NHS Digital. (2009). Adult psychiatric morbidity in england - 2007, results of a household survey. Retrieved from https://digital.nhs.uk/data-andinformation/publications/statistical/adult-psychiatric-morbidity-survey/adult-psychiatricmorbidity-in-england-2007-results-of-a-household-survey

Pesola, F., Shelton, K. H., \& van den Bree, Marianne BM. (2014). Sexual orientation and alcohol problem use among UK adolescents: An indirect link through depressed mood. Addiction, 109(7), 1072-1080.

Pilleron, S., Guerchet, M., Ndamba-Bandzouzi, B., Mbelesso, P., Dartigues, J., Preux, P., .. . EPIDEMCA Group. (2015). Association between stressful life events and cognitive disorders in central africa: Results from the EPIDEMCA program. Neuroepidemiology, 44(2), 99-107.

R Foundation for Statistical Computing, Vienna, Austria. (2019). R: A language and environment for statistical computing. Retrieved from https://www.R-project.org/

Rourke, S. B., Halman, M. H., \& Bassel, C. (1999). Neurocognitive complaints in HIVinfection and their relationship to depressive symptoms and neuropsychological functioning. Journal of Clinical and Experimental Neuropsychology, 21(6), 737-756.

Sabia, S., Elbaz, A., Dugravot, A., Head, J., Shipley, M., Hagger-Johnson, G., . . . SinghManoux, A. (2012). Impact of smoking on cognitive decline in early old age: The whitehall II cohort study. Archives of General Psychiatry, 69(6), 627-635.

Saunders, J. B., Aasland, O. G., Babor, T. F., De la Fuente, Juan R, \& Grant, M. (1993). Development of the alcohol use disorders identification test (AUDIT): WHO 
collaborative project on early detection of persons with harmful alcohol consumption-II. Addiction, 88(6), 791-804.

Semlyen, J., King, M., Varney, J., \& Hagger-Johnson, G. (2016). Sexual orientation and symptoms of common mental disorder or low wellbeing: Combined meta-analysis of 12 UK population health surveys. BMC Psychiatry, 16(1), 67.

Smith, L., Jacob, L., Yakkundi, A., McDermott, D., Armstrong, N. C., Barnett, Y., . . Tully, M. A. (2020). Correlates of symptoms of anxiety and depression and mental wellbeing associated with COVID-19: A cross-sectional study of UK-based respondents. Psychiatry Research, 291, 113138.

Stockwell, T., Sitharthan, T., McGRATH, D., \& Lang, E. (1994). The measurement of alcohol dependence and impaired control in community samples. Addiction, 89(2), 167184.

Toyoshima, K., Inoue, T., Shimura, A., Masuya, J., Ichiki, M., Fujimura, Y., \& Kusumi, I. (2020). Associations between the depressive symptoms, subjective cognitive function, and presenteeism of japanese adult workers: A cross-sectional survey study. BioPsychoSocial Medicine, 14, 1-7.

Tsapanou, A., Vlachos, G. S., Cosentino, S., Gu, Y., Manly, J. J., Brickman, A. M., . . . Kosmidis, M. H. (2019). Sleep and subjective cognitive decline in cognitively healthy elderly: Results from two cohorts. Journal of Sleep Research, 28(5), e12759. 